\title{
Die Zeitschrift für öffentliche und gemeinwirtschaft- liche Unternehmen als Organ des BVÖD
}

Seit 1985 war die Zeitschrift für öffentliche und gemeinwirtschaftliche Unternehmen (ZögU) als unabhängige wissenschaftliche Fachzeitschrift zugleich Organ der Gesellschaft für öffentliche Wirtschaft (GÖW). Zum 31. Dezember 2007 ist die Gesellschaft für öffentliche Wirtschaft aufgelöst worden. Ihre wissenschaftlichen Aufgaben auf dem Gebiet der öffentlichen und gemeinwirtschaftlichen Dienstleistungen werden künftig im „Bundesverband Öffentliche Dienstleistungen - Deutsche Sektion des CEEP“ (BVÖD) wahrgenommen.

Der Bundesverband Öffentliche Dienstleistungen ist im Mai 2007 durch Umgründung der Deutschen Sektion des Europäischen Zentralverbandes der öffentlichen Wirtschaft (CEEP) in einen eingetragenen Verein entstanden. Er nimmt im europäischen Rahmen über den CEEP die Berufsverbandsfunktion für die Unternehmen, die in Deutschland öffentliche Dienstleistungen - in der EU-Terminologie: Dienstleistungen von allgemeinem bzw. allgemeinem wirtschaftlichen Interesse - erbringen, sowie die Sozialpartnerfunktion für die deutschen öffentlichen Arbeitgeber wahr. Für die neu hinzugekommene wissenschaftliche Aufgabenstellung steht dem Bundesverband Öffentliche Dienstleistungen der bisherige Wissenschaftliche Beirat der Gesellschaft für öffentliche Wirtschaft zur Seite.

Wie zuvor der Gesellschaft für öffentliche Wirtschaft wird die ZögU künftig dem Bundesverband Öffentliche Dienstleistungen als Organ dienen. Die ZögU wird auch in Zukunft ihren wissenschaftlichen Charakter beibehalten. Das neue - vergrößerte und verjüngte - Herausgeber-Team, das sich durch hohe wissenschaftliche Qualifikation auszeichnet, wird mit Professor Frank Schulz-Nieswandt an der Spitze hinsichtlich des Inhalts der Zeitschrift dieselbe Unabhängigkeit besitzen wie die Herausgeber der ZögU in der Vergangenheit. Dies ist niedergelegt in einem Vertrag, den der BVÖD mit der Nomos Verlagsgesellschaft und den Herausgebern geschlossen hat. Die eingereichten wissenschaftlichen Fachbeiträge werden wie bisher einem strengen Referee-Verfahren unterworfen sein, und die Herausgeber werden auch künftig von einem Beirat - jetzt als Herausgeberbeirat bezeichnet - unterstützt und beraten werden.

Berlin, März 2008

\section{Hans-Joachim Reck}

Hauptgeschäftsführer des Verbandes kommunaler Unternehmen

Präsident des Bundesverbandes Öffentliche Dienstleistungen - Deutsche Sektion des CEEP 